\title{
EXTRAVAGANCES IN THE MOTOR THEORIES OF CONSCIOUSNESS
}

\author{
BY H. C. McCOMAS
}

Princeton Universily

The biological point of view in psychology has brought the so-called motor theories of consciousness into sharp relief. As yet there has been no formulation of these theories into a coherent whole. They represent a tendency in the thinking of today and not a consensus of opinion. The disposition to interpret consciousness in motor terms is not new; it may be found in many of the earlier writers of modern psychology who sought explanations in the motor factors for various aspects of consciousness, such as attention, space-perception, ideo-motor actions, emotion, and volition. Today the more ambitious program would include all aspects of consciousness. Broadly stated, all consciousness is conditioned or accompanied by motor activity. Such assumptions may be detected in much of the recent work in psychology. It is difficult, however, to find completely rounded out theories. Of these the most influential are probably those of Dewey, Mūnsterberg, Judd, and Watson.

Chronologically Dewey ${ }^{1}$ stands in the forefront. His conception of the reflex arc as converted into a circuit laid great stress on the motor side of processes that were commonly considered sensory. The sensory-motor processes are all one process in this scheme. "It is just as true to say that the sensation of sound arises from a motor response as that the running away is a response to the sound." The reason for this lies in the fact that "the sound is not a mere stimulus or mere sensation; it again is an act, that of hearing. The muscular response is involved in this as well as sensory stimulus. . . . The movement and posture of the head, the tension of the ear

$1 \mathrm{~J}$. Dewey, 'The Reflex Arc, Concept in Psychology;' Psrcror. Rzv., 1896, 3, $357-370$. 
muscles, are required for the reception of the sound. ... The conscious sensation of sound depends upon the motor response having already taken place. . . . Indeed, the movement is only for the sake of determining the stimulus, of fixing what kind of stimulus it is, of interpreting it."

Dewey's concept holds sensory, central, and motor processes as coördinations of one process. This would certainly imply that the motor elements were indispensable in consciousness.

Münsterberg's' 'Action Theory' maintains that every sensation and every element of consciousness is dependent on the passage of stimulation into discharge in the cortex of the brain. The quality, intensity, and vividness of sensations depend upon the parts of the cortex affected and the character of the current passing through to the motor tracts. In a recent formulation of his theory ${ }^{2}$ he declares that whether a color or a noise become vivid in the mind or are refused admittance depends upon the conditions which prevail in the motor centers. If the channels of discharge are closed the sensations will be inhibited. "According to the popular view a world of impressions and ideas exists in us, entirely independent of our actions; and when they are complete and perfect they send their message to some motor apparatus which carries out the order. Such a fancy must be entirely reversed. In every moment the motor situation decides the possibilities in the sensory sphere. Our ideas are the product of our readiness to act." Whether the author means ready to act or action is not clear, for in another place ${ }^{3}$ he declares: "The centripital function appears to be almost more important than the centrifugal one which bears the stimulus. It is the action of the organism which controls the mental life." It would seem from the following that an actual movement were necessary: "The personality's thinking is as much the product of his actions as his actions are the product of his thought."

In Judd's psychology the motor theory is everywhere

1 H. Münsterberg, 'Grundzüge der Psychologie,' Bd. I., S. 548.

2 'Psychology, General and Applied,' 139-144.

Op. cit., 423 .

'C. H. Judd, 'Psychology,' 71, 134, 296, 183. 
present. In an early chapter he states, when analyzing consciousness: "In some form or other, every incoming sensory impulse and every central nervous process must issue in a motor discharge. It is equally true, though by no means so obvious, that every form of conscious experience is related to behavior." In discussing sensations he says: "Our problem is not exhausted, however, when we have dealt with the interrelation between sensations, for every sensation is related in some way to the active processes of expression." In the complex associational processes there may be a delay of minutes, or days, before the excitation is "discharged through the motor centers in the form of a reaction." His conviction that "there is a universal relation between consciousness and bodily activity," rests upon biological grounds. "The structure of the nervous system from the hydra to man is such that there is always a motor organ linked with every sensory organ."

With Watson ${ }^{1}$ we come to the logical culmination of this trend of thinking. "The nervous system functions in complete arcs. An incoming impulse exerts its effect relatively immediately upon one system of effectors or another." So precise is this relation that, "in a system of psychology completely worked out, given the responses the stimuli can be predicted; given the stimuli the responses can be predicted." The responses may be 'explicit' or 'implicit'; in the latter case they involve chiefly the actions of speech mechanisms. If these could be detected and recorded the material which the introspectionist claims as peculiarly his own would be accessible to the behaviorist, and the thought processes would be interpretable in terms of their motor accompaniments. So confident is Watson in the principle of the complete arc functioning in thought and speech that he declares: "If we could find a case where a man suddenly lost his laryngeal apparatus without any serious injury to the bodily mechanisms, we should have a crucial case. From our point of view there would, or ought to be, a serious limitation in this man's thought processes."2 These brief citations do not do justice to the author's

IJ. B. Watson, 'Behavior; an Introduction to Comparative Psychology,' Chap. 1 .

2 Ibid., 327. 
theories in their entirety; that would be impossible in a short article. Nor is it the present purpose to discuss these theories. The issue in hand is to point out an obvious extravagance running through this trend of thinking; namely, the insistence that a motor expression accompanies all conscious processes. No one will deny that there is a deep-seated tendency for the incoming impressions to go out into motor expressions; but there is nothing more than a tendency. The authors quoted above, and a number of others who have written less fully and frankly, push this principle to an extreme. They are seeking to make it an open-sesame into the most intricate problems in psychology.

To put the most obvious criticism into logical form, the champions of the motor theories commit a fallacy in generalization. They infer of a whole class what we know only of a part.

Moreover, they are captivated by the principle of continuity. The structure and the function of the nervous system in its evolution do undoubtedly show the intimate relation of receptor and effector, of stimulus and response. It is very easy to assume that a principle which is corroborated so frequently in the animal scale should apply to the most intricate type of nervous system. But does it? Facts alone can answer the question.

The influence of biology is clearly discernible in these theories. The psychologist who thinks of the nervous system in terms of the hydra may readily imagine every stimulus effecting a prompt response, but one who thinks in terms of human anatomy can hardly do so, for the anatomist himself is too circumspect. Villiger, ${ }^{1}$ for example, insists that "only after traversing numerous neurones does the impulse finally reach the motor center and from there pass to the motor path, since coöperation of the various cortical centers must be assumed in explanation of the complex psychic processes." If this passage were by simple nerve trunk conduction every stimulus might go out into a response, but it is not so. A series of obstinate neurones and synapses must be traversed in the cortex and an excitation may die out anywhere along the

${ }^{1}$ E. Villiger, 'Brain and Spinal Cord,' 133. 
line. Sherrington ${ }^{1}$ shows that the reflex chain of several neurones offers higher resistance than that of a few neurones. What would happen if an impulse stalled in some high-threshold synapse before reaching a motor tract? Nothing, says the motorist; it must cross the mystic line (Münsterberg); it must make the round trip (Watson). Only when it crosses the frontier and enters the motor tract is it given a passport into consciousness.

If this be so, then the motor areas are most important for consciousness. Injuries in them should do greater damage to consciousness than injuries elsewhere. Southard ${ }^{2}$ finds the reverse to be true. Injuries in the posterior areas cause greater disturbances than those in the anterior. Cushing found that stimulating sensory areas did give an effect in consciousness, but the same stimulation in motor areas did not. Even if we ignore the cortex and consider the structure of the afferent and efferent tracts we can find nothing to support the motorist's ideal. According to his schema the two sets of tracts have equal tasks, they are parts of one circuit, or process, the motor being as active and important as the sensory. Anatomically, nothing of the kind exists. The sensory tracts outnumber the motor by three or four to one, and there are more neurones in the former than the latter. Moreover, when we compare the complexity of the sense organs with the simplicity of the muscles and glands the disparity is overwhelming. Washburn's contention that "every sensation that can be discriminated in a fusion, and every group of sensations that can be attended as a single whole has connected with it one or more movements which are peculiar to it alone," calls for a much more elaborate motor apparatus than nature supplies. It is inconceivable that each fiber in the basilar membrane, affording over twenty thousand discriminable tones, should have its accompanying little movement with a meaning all its own. Indeed, if any inference is to be drawn from the structure of the human animal it must give a greater prominence and

${ }^{1}$ C. S. Sherrington, 'The Integrative Action of the Nervous System,' p. 156.

2 E. E. Southard, Psychol. Bull., 1914, xx, 66.

${ }^{2} \mathrm{H}$. Cushing, Brain, 1909, 32, 44-53.

- 'The Function of Incipient Motor Proresses,' Psychol. Rev., 1914, 21, 376-390. 
importance to the sensory organs, tracts, and areas than to the motor. The two systems are not on a parity. Nothing but the exigencies of a theory could assign them equal duties in conscious processes.

Nor are the motor theories in better case when we turn to experimental results for evidence of invariable response to stimuli. Truly, many organic changes accompany various conscious states, but a close correspondence of the one to the other is far from proved. Shepard ${ }^{1}$ in summarizing the results of work in this field says: "No factor, vasomotor, rate or amplitude of pulse, position or emphasis of dicrotic, or rate or amplitude of breathing, changes one way for agreeable and the opposite way for disagreeable conditions. ... The rate of the heart is sometimes increased on the average, sometimes decreased, sometimes not changed at all. . . The rate of the heart with sensory stimuli increases about as often as it decreases. Mental application gave a slight amount of vasomotor change, frequently so slight as to be hardly noticeable. ... In about half of the mental application tests there are both vasodilation and vasoconstriction in a single test. When the change is only in one direction it is an even chance that it be dilation or constriction." Surely, no one would venture to predict the stimuli, given the responses, in that mêlée of confusions; and only the hardiest of theorists would seek to demonstrate how the organic movements determine the conscious states from that material.

The experimental results bearing upon the relation of unconscious movements to impressions and ideas are also inadequate to the motorist requirements. Stein ${ }^{2}$ found that it was possible to educate many subjects to make automatic movements. However, there was a large percentage of both males and females from whom she could get no automatic movements at all. The so-called ideomotor reactions are nothing more than habits. They do not rest upon any physiological principle similar to that of reflexes, or instincts. They grow up out of experiences as do all associations. As we

1 Amer. J. of Psychal., 1906, 17, 522-584.

2 Psychol. Rev, 1898, 5, 295-306. 
habitually associate the words 'cat' and 'dog,' 'pretty' and 'girl,' so too do we associate an oncoming baseball and the catcher's movement, or the threatened blow and the pugilist's 'on guard.' Many of us accompany our language with gestures. These easily become habitual and accompany certain thoughts which may or may not be expressed in words. This conception of ideomotor action is supported by a study of the involuntary movements of children and adults. ${ }^{1}$ Among children there is less directness in such movements than in adults. In imitating unconsciously the movement of a moving object they are less susceptible than adults. They show less fixity than adults in both their movement and verbal associations. It is habit, extending over a period of years, that gives permanency to associations. There is nothing in the oft-quoted ideomotor actions to warrant the assumption that every incoming impression goes at once into some form of movement, and that this is based upon the structure of the nervous system.

In speech-motor reactions it is probable that the same habit principle obtains. Curtis ${ }^{2}$ fastened a tambour to the larynx in such a way as to record slight movements there. He could obtain records when his subjects read silently or recited mentally. Nevertheless, twenty per cent. of his subjects gave no records of any movements.

The motorist is not on safe ground when he retreats to the position that impressions eventually issue into action. This assumes that an impression may persist for a long time and then assert its motor rights. Some impressions may do that. Many of them fade out, merge into a type or into some other impression. We can no longer hold the fantastic belief that every impression leaves an indelible mark on the soul. The few striking instances of memories evoked in delirium, or hypnosis, which bring forth events forgotten for years, do not prove a miraculous retention on the part of the cortex. If every impression goes forth into expression, then each tree, twig, stone, pebble, sparrow, and cloud that flits past the car window from Boston to San Francisco will leave a mark on the obser-

${ }^{1} \mathrm{M}$. A. Tucker, 'Comparative Observations on the Involuntary Movements of Adults and Children,' Amer. J. of Psychol., 1896-97, 8, 394-404.

Amer. J. of Psychol., 1900, 11, 237-239. 
ver's nervous system which will later issue in some form of behavior! This must happen despite the fact that the observer forgets these trifles as quickly as he sees them!

A less fantastic and more important feature of the motorist's program seeks to maintain the doctrine that conscious states are conditioned, or determined, by motor activities. This does not mean that the kinesthetic sensations of movements accompanying perceptions blend with these perceptions and give a certain character to them. That is an old, an obvious fact. The conception is derived from the belief that sensory, central, and motor processes are one process. All three function together. It is an extreme statement of the unity of the nervous system and of conscious activities. Its over-emphasis distorts a valuable truth and a serviceable conception.

The strongest arguments for the contention are to be found in the relations between the thought and speech processes. So intimately is speech bound up with ideation and, indeed, perception that it would seem impossible to separate them. If the motor discharge conditions the conscious content in any activity it must do it here. If the motor areas are indispensable to the neural basis of any conscious state, they are indispensable here. This is so apparent that Watson pins his faith to the integrity of the process. A man lacking speechmotor action is a 'crucial case' for behaviorism. Watson believes that such cases are 'extremely unlikely' to occur; they are rare, but they do occur. Pure motor aphasia calls for a disorder in such a minute and restricted area that few cases are recorded. Usually the disturbance involves other parts and a number of sensory and motor defects accompany the aphasia. Rare though such cases are, they are sufficiently well known and adequately described for the purpose in hand. Dejerine" states that "the sole phenomenon consists in the impossibility of the articulation of sounds in all their modes, but all other qualities of language are intact and the interior language functionates as in the normal individual." Moutier says the condition is one in which the patient finds it impossible to communicate with his entourage, the tongue does not obey

${ }^{1}$ Quoted by Dercum, $J$. of Nero. and Ment. DAsease, 1914, 41, 137-141. 
the will. The thoughts in his mind are very clear and his words precise, but he cannot exteriorize them at all. "There is present but one symptom and that is the suppression of articulate language." Dercum describes such a case. His patient showed no sensory losses at all. "He had apparently complete motor aphasia. He was unable to make the slightest articulate sound. . . . At the same time the patient's understanding of spoken speech and comprehension of what was said to him was perfect. . . . Speech comprehension was completely preserved. Further, the patient could read written or printed matter and carry out instructions perfectly." There was no agraphia, he could write spontaneously and from dictation. Dr. Dana ${ }^{1}$ reports three cases on his lists. Of one he says: "The patient cannot say a word, or repeat, or ejaculate, or count. But he writes, reads, understands, and has good general intelligence." In short, here we have several cases of the loss of the speech mechanisms and no 'serious limitation' in the patient's 'thought processes.' The elimina, tion of the motor factor has not disturbed the central and sensory processes. The perception of words has not been impaired, much less inhibited, by the inability of the incoming impulse to discharge into the speech motor area.

If the motor discharge into the larger musculature of the trunk and limbs were necessary to normal conscious life we should expect to find curious distortions in the thought and feelings of anyone suffering from a motor paralysis. Indeedfrom the certainty which runs through the motor theories, we should be able to map out definite fields of consciousness which would be impaired as a paralysis spreads over legs, trunk and arms. As a matter of fact nothing of the kind occurs. Cases may be found where only the spinal motor tracts are involved. In these there is no obliteration of large parts of the thought and emotional life of the patients-certainly no impairment of perception.

It seems probable that the line of attack will be shifted by the more ardent of the motorists from overt movements of the musculature to less accessible regions, where observation

1 N. Y. Med. Journal, Aug. 10, 1907. 
and experiment cannot follow. The glands offer a tempting opportunity. If we must have every incoming impulse and every central nervous process issuing in a motor discharge, why not assign the splendid duty to that famous and historic organ, the pineal gland? 